\title{
Erratum: Hidden momentum and the Abraham-Minkowski debate [Phys. Rev. A 95, 043804 (2017)]
}

Pablo L. Saldanha and J. S. Oliveira Filho

(Received 9 July 2018; published 23 July 2018)

DOI: 10.1103/PhysRevA.98.019903

In our paper there was a mistake on the expression for the electromagnetic momentum density of a medium composed of neutral particles whose electric dipole moments $\mathbf{p}_{i}$ are the result of magnetic currents, and the magnetic dipole moments $\boldsymbol{\mu}_{i}$ are the result of electric currents. The last sentence of the paragraph that begins on p. 3 and ends on p. 4 should be "The electromagnetic momentum density in this case becomes $\mathbf{E} \times \mathbf{H} / c^{2}-\mathbf{M} \times \mathbf{E} / c^{2}+\mathbf{P} \times \mathbf{B}=\mathbf{D} \times \mathbf{B}$, which is the Minkowski momentum density." The expression $\mathbf{D} \times \mathbf{B}$ should also be the electromagnetic momentum density written on the corresponding line of Table I. The correct table is shown below.

TABLE I. Association of different models for the electric dipoles $\mathbf{p}_{i}$ and magnetic dipoles $\boldsymbol{\mu}_{i}$ of the particles that compose the medium with the corresponding expressions for the electromagnetic momentum density.

\begin{tabular}{llcr}
\hline \hline Model for $\mathbf{p}_{i}$ & Model for $\boldsymbol{\mu}_{i}$ & Hidden momentum density & Electromagnetic momentum density \\
\hline Electric charges & Magnetic charges & 0 & $\mathbf{E} \times \mathbf{H} / c^{2}$ \\
Electric charges & Electric currents & $\mathbf{M} \times \mathbf{E} / c^{2}$ & $\varepsilon_{0} \mathbf{E} \times \mathbf{B}$ \\
Magnetic currents & Electric currents & $-\mathbf{P} \times \mathbf{B}+\mathbf{M} \times \mathbf{E} / c^{2}$ & $\mathbf{D} \times \mathbf{B}$ \\
Magnetic currents & Magnetic charges & $-\mathbf{P} \times \mathbf{B}$ & $\mathbf{E} \times \mathbf{H} / c^{2}+\mathbf{P} \times \mathbf{B}$ \\
\hline \hline
\end{tabular}

